\title{
Features of current-voltage characteristics inherent to GaP light-emitting diodes with quantum wells
}

\author{
O. Konoreva ${ }^{1}$, V. Opilat ${ }^{2}$ M. Pinkovska ${ }^{1}$, V. Tartachnyk ${ }^{1}$ \\ ${ }^{I}$ Institute for Nuclear Research, NAS of Ukraine, 47, prospect Nauky, 03028 Kyiv, Ukraine \\ ${ }^{2}$ Drahomanov National Pedagogical University, 9, Pirohova str., 01601 Kyiv, Ukraine \\ Corresponding author: phone: +044-525-37-49, fax: +044-525-44-63 \\ E-mail:myrglory@yahoo.com; opylat@ua.fm
}

\begin{abstract}
In this work, GaP $p$ - $n$ junctions used in light-diode manufacturing were studied using the electrophysical methods at various temperatures. Current-voltage characteristics of some diodes, controlled by PC and measured in the voltage and current generator modes with various steps, have shown irregularities in the regions of negative differential resistance and specific before-breakdown part. Long-lasting relaxation of conductivity of GaP crystal with nonuniformity of defect distribution was observed. From analysis of current-flow mechanisms, it was proposed that atypical GaP light-diode electrical characteristics degradation is caused by complex traps shaped as quantum wells in the $p-n$ junction.
\end{abstract}

Keywords: GaP, current-voltage characteristics, irregularities, complex traps, quantum wells, conductivity relaxation.

Manuscript received 23.05.06; accepted for publication 23.10.06.

\section{Introduction}

Stability of element base parameters of modern solidstate radioelectronic devices is mainly determined by the quality of their active regions: $p-n$ junctions, heterostructures, and metal-semiconductor junctions. And the perfection of semiconductor interface layers used in a micromodule device fabrication is a problem, especially, for such material as GaP. For example, nearly thirty years ago it was discovered using the positron annihilation method that the $\mathrm{GaP}$ film-substrate interface was enriched by vacancies, and $200{ }^{\circ} \mathrm{C}$ annealing tended to polivacancy cluster creation [1]. The high density of dislocations that can grow at the whole upper layer depth and thus influence on the device characteristics was obtained in [1-4]. Different aspects of defect structure of $\mathrm{GaP}$ exposed to ionizing radiation, are studied till now (see for example [5-7]). But knowledge of the defectness of $p$ - $n$ junction as a prerequisite of light-diode operating characteristics is still desirable.

In this article, the defect states of $\mathrm{CZ} \mathrm{GaP} p$ - $n$ junctions used in light-diode fabrication were studied using the electrophysical methods (conductivity, capacity-voltage and current-voltage dependences). This makes it possible to study mechanisms and processes that occur in the $p-n$ junction. The main attention was paid to the appearance of irregularities in current-voltage characteristics, especially the regions with negative differential resistance and breakdown. In some devices the observed anomalies of electrical characteristics and long-lasting conductivity relaxation are connected with uniform distribution of impurities and existence of complex traps shaped as quantum wells in the depletion region of $p-n$ junction.

\section{Experiment}

Fabricated GaP light diodes made by the double liquidepitaxy method were used. $p-n$ structures with red emission were doped by $\mathrm{Zn}$ and $\mathrm{O}$ atoms, and with green - by $\mathrm{N}$ atoms. Conductivity of cross-like $\mathrm{GaP}$ samples was measured as a time function at the room temperature after thermal excitation. The $p-n$ junction parameters were obtained from capacity-voltage measurements. Currentvoltage characteristics (CurVC) of light diodes controlled by $\mathrm{PC}$ in the voltage and current generator modes were studied at the nitrogen and room temperatures. The opportunity to change current or voltage steps makes it possible nearly to instantly make measurements and thus to observe a thin structure in CurVC of GaP $p$ - $n$ junction. 


\section{Results and discussion}

Changes of conductivity in GaP samples last after thermal excitation for several dozen of minutes at room temperature (Fig. 1). As shown in [8-10], relaxation curves $\sigma(t)$ are formed in crystals that possess nonuniform impurity distribution. In order to explain the relaxation mechanism, the band scheme that included defect clusters (shown in the insert in Fig. 1) was used. It makes it possible to estimate the main cluster parameters, namely: the potential barrier height and geometric dimensions.

Equations that describe the barrier height $\varphi_{\text {eff }}$ and effective cluster radius $R_{\text {eff }}$ are [8]:

$\varphi_{\text {eff }}=\frac{\hbar e}{\varepsilon R_{e f f}}$,

$R_{\mathrm{eff}}=\frac{\hbar^{2} e^{2} \eta}{8 m^{*} \varepsilon k^{2} T^{2}}$,

where $\eta$ is the defect concentration in a cluster, $e, \hbar, \varepsilon, T$, $k$ are the well-known physical parameters.

Using the well-known relations for the timedependent conductivity

$\sigma(t)=\sigma_{\infty}+\sigma_{0} \exp \left(-\frac{t}{\tau}\right)$

and lifetime

$\tau=\tau_{0} \exp \left(\frac{\varphi_{\text {eff }} e}{k T}\right)$

from the experimental data, one can estimate the defect concentration in a cluster, its effective radius and potential barrier around it.

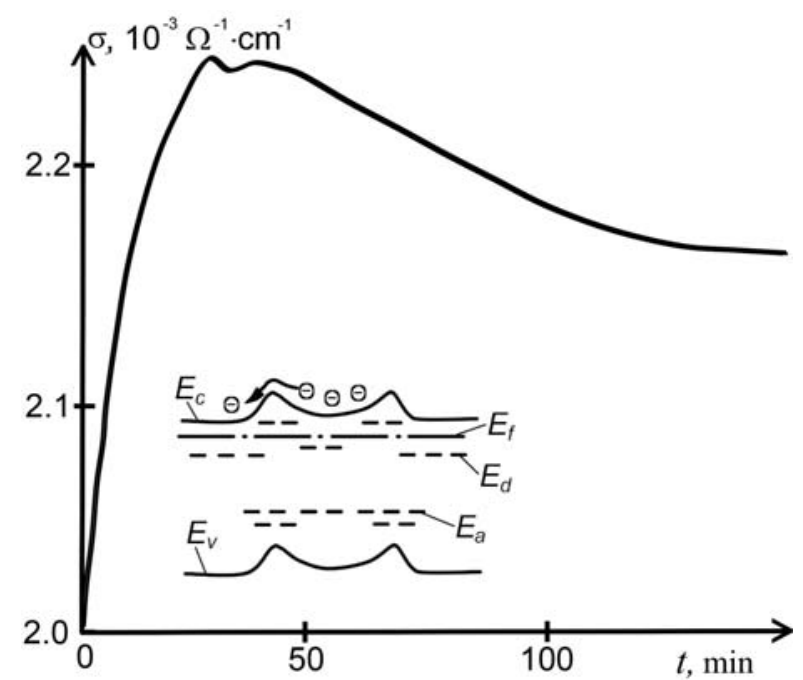

Fig. 1. Time-dependent conductivity relaxation of GaP crystal at the room temperature. Band scheme that includes defect clusters is shown in the insert.
As relaxation processes proceed long enough (some dozens of minutes), the calculated from Eq. (1) potential barrier is essential, it equals nearly $0.7-0.9 \mathrm{eV}$. The effective cluster radius of about 40-60 $\AA$ (Eq. (2)) responds to it.

Forward CurVC of some red and green GaP lightdiodes, made of such crystal and used in investigations, possess anomalies. $I-U$ curves of diodes measured with low current steps display separate oscillations at 70-90 K (Fig. 2) and a characteristic negative differential resistance region (NDR) is observed just before this region. The origin and main details concerning the current flow in NDR were studied previously in detail [11].

At reverse bias, the situation is quite different. Some light diodes measured at the room temperature for 4 times, display atypical reverse $I-U$ dependences too, and the current curve shape depends upon the number of the measurement (Fig. 3). One can see that the measured for the first time reverse $I-U$ dependence is characterized by the minimum (curve 1), and its deepness decreases with repeating the measurements. At last after 4 cycles, the current curve shape has become similar to that obtained at $77 \mathrm{~K}$. It was stated experimentally that to reach again a reproduction of the reverse $I-U$ curve with the minimum (after 4 measurements and obtaining the smooth curve), it was necessary to keep the sample at the nitrogen temperature for $10 \mathrm{~min}$. The nature of current recovering needs an additional investigation.

It is worthy to mention once more that, as a rule, the experimental curves correspond to ideal $p$ - $n$ junction characteristics, and only in some investigations the current curve possesses minimum.

In a contrast with the previous case $(300 \mathrm{~K})$, the reverse $I-U$ dependence at $77 \mathrm{~K}$ is typical for $p-n$ junction and is caused by minority carrier flow that is

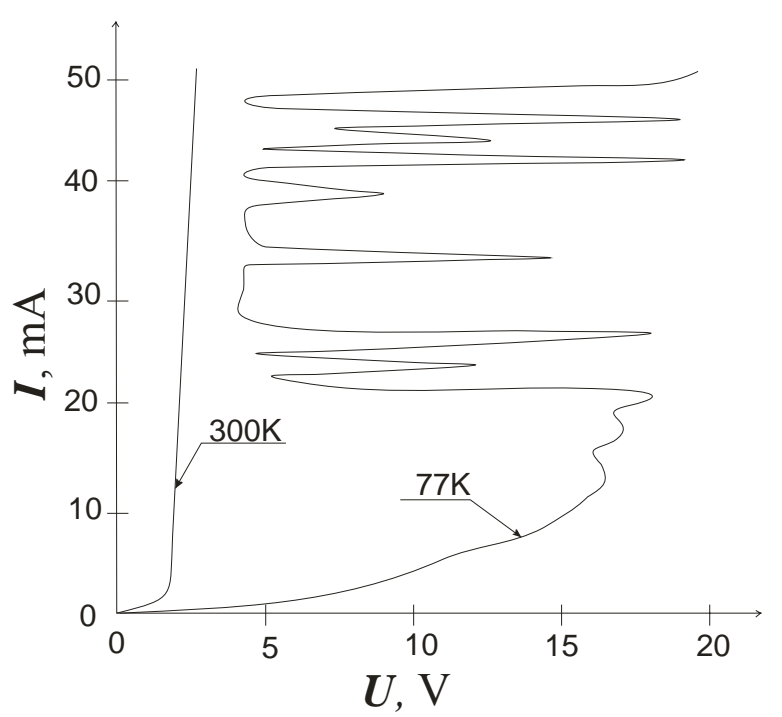

Fig. 2. Current-voltage characteristics of GaP light-emitting diode measured at various temperatures. 
followed by the breakdown region. This curve nearly coincides with the room temperature reverse characteristics, obtained for the last measurement of cycle (see curve 4 in Fig. 3). The first part of the curve is practically temperature independent till starting the breakdown.

Let us analyze a possible mechanism of the atypical $I-U$ dependences. Thermal $p-n$ junction breakdown is caused by the conductivity increase with temperature. So, it is difficult to explain the negative resistance region with minimum changing during every cycle. The avalanche breakdown is characterized by a sharp (nearvertical) current dependence on the voltage. So, one can suppose large-dimension structure defects that provoked long-lasting relaxation processes influence on the atypical reverse current. As shown previously by studying conductivity relaxation in GaP, clusters with radius close to several dozens of angstroms can exist in crystal used for light-emitting diode manufacturing. If such defects are shaped as the wells, surrounded by barriers, as shown in the insert in Fig. 1, the wells can store the current carriers and then return them (under specific conditions) to the conduction or valence bands. In the case, the defects are in the depleted diode region, the $p-n$ junction field changes unequally barrier height from the both well sides: namely, by increasing a barrier height from the one side, and decreasing - from another. Thus, the conditions are created for over-barrier emission and for the increase of the tunneling probability (see Fig. 4).

From the CurVC measurements, one can evaluate the maximum field $E$ in a $p-n$ junction [12]:

$$
E=\frac{z\left(U+U_{k}\right)}{\varepsilon S(z-1)} C \text {. }
$$

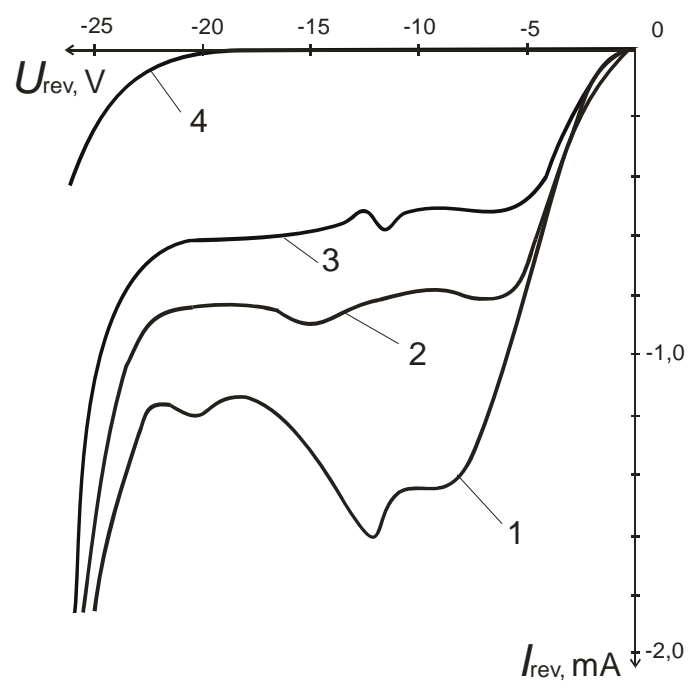

Fig. 3. Atypical GaP diode current-voltage characteristics measured repeatedly (curves 1-4) at $300 \mathrm{~K}$. The latter curve 4 coincides with the characteristics measured at $77 \mathrm{~K}$.

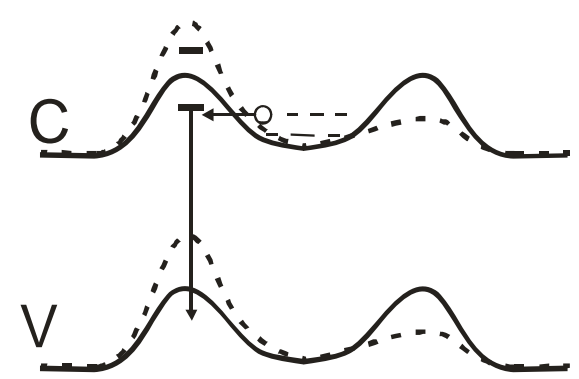

Fig. 4. Schematic band structure of the initial crystal with impurities, shaped as "quantum wells".

It follows from the $I-U$ curve slope $z=2 . U_{k}$ contact potential, $U-$ reverse voltage. The diode parameters: area $S=1 \mathrm{~mm}^{2}$, barrier capacity $C=60 \mathrm{pf}$. Then $E=3.6 \cdot 10^{6} \mathrm{~V} / \mathrm{cm}$.

When the reverse bias reaches the value, at which the minimum in the $I-U$ curve occurs $\left(U_{r}=10 \mathrm{~V}\right)$,

$E(10 \mathrm{~V})=9.72 \cdot 10^{6}(\mathrm{~V} / \mathrm{m})$.

Initial field of the cluster surrounded by the barrier, which was derived from the abovementioned assumption (concerning the barrier potential $\varphi_{\text {eff }}=0.6 \mathrm{eV}$ and radius $r=60 \AA$ ) is close to the same value. So, the tunneling threshold $U_{r}=10 \mathrm{~V}$ [13] for GaP diode is overcame. Then, the carrier tunneling through the barrier dominates in the part where the current increases to the value $I_{\min }$. Unfortunately, we can't confirm the current mechanism as the tunneling using the slope of current dependence on temperature, because the value of the current minimum is changed with repeating the measurements.

Appearance of complex defects shaped as "quantum wells" may be caused by nonhomogeneities of the impurity distribution in the initial sample. One can assume that a local center is situated on the back side of the central well (shown schematically in Fig. 1). At a large electrical field, electrons from the filled central part move through a barrier to the local center with the following transit to the valence band. The reverse current increases as the bias increases. If the bias is too large and the defect level position shifts enough, the transit of the well-defect has become impossible (dotted line in Fig. 4). The diode reverse current decreases to the level of saturation.

An existence of structure defects of quantum well shape, surrounded by three-dimensional potential barriers, looks like sample memory, caused by manufacturing operations. Filling of centers by carriers may occur due to over-barrier emission at hightemperature treatment. This state can be storied long enough, because the barrier height at $300 \mathrm{~K}$ is greater than $k T$ value. Such effect is quite similar to that observed in the information storage by TLD dosimeter.

Analogous quantum wells are situated randomly in a crystal. Their influence on reverse currents of $p-n$ junction is effective only in the case, when some of the 
wells appear in the depletion region of the $p$ - $n$ junction. If the wells are far from the region of the maximum electrical field, the current-voltage dependences possess no anomalies.

The decrease of minimum of $I_{r}\left(U_{r}\right)$ dependence with every cycle repeating is probably caused by large junction potential barriers and large relaxation times: potential wells are not emptied fully in the course of measurements.

The sharp increase of $I_{r}$ curve at a high voltage is caused by the carrier tunneling, as the slope of $I-U$ curves is the same at 300 and $77 \mathrm{~K}$. Tunneling breakdown occurs at the voltages $e\left(U_{r}+U_{k}\right)<E_{g}$, when the band shift allows effective band-to-band tunneling [14] with the tunnel probability $D$ according to the equation

$$
D=\exp \left(-\frac{4}{3} \sqrt{2 m^{*}} \frac{E_{g}^{3 / 2}}{\hbar q W_{e}}\right),
$$

where $W_{e}$ is the current carrier energy, $E_{g}$ is the forbidden band width, $m^{*}, \hbar, q$ are the crystal parameters.

\section{Conclusion}

It has been found the long-lasting relaxation of conductivity in GaP samples with non-uniformity of the impurity distribution. Processes are caused by the existence of complex traps shaped as quantum wells with several dozens of Angstroem unit dimensions, which are surrounded by potential barriers with the barrier height equal to $\sim 0.6 \mathrm{eV}$.

Current anomalies were observed in some red and green GaP CurVCs. The region of the sharp reverse current increase is obvious for the $I-U$ dependences at $300 \mathrm{~K}$, which is followed by negative differential resistance. The effect is caused by the carrier tunneling from the quantum well onto the local defect (located in potential barrier boundaries) and following emission into the valence band. The quantum wells influence on $p-n$ junction reverse currents only in the case, when they are situated in the depletion region of the $p-n$ junction. If wells are far from the region of the maximum electrical field, the current-voltage dependences possess no anomalies.

\section{References}

1. O.F. Nemets, N.E. Grigorian, P.G. Litovchenko, V.Ja. Opilat, V.P. Tartachnyk, I.I. Tychyna, Radiation defects in GaP, irradiated by $\alpha$-particles and electrons // Doklady AN USSR, Ser.A, 8, p. 5456 (1988) (in Russian).
2. D.N. Shaw, Influence of substrate temperature on GaAs epitaxial deposition rates // Electrochem. Soc. 115, p. 405 (1968).

3. R.A. Burmeister, G.P. Pighini, P.E. Greene, Large area epitaxial growth of $\mathrm{GaAs}_{1-\mathrm{x}} \mathrm{P}_{\mathrm{x}}$ for display applications // Trans. Metal. Soc. AYME 245, p. 587-593 (1969).

4. G.A. Rozgonoi, R.H. Saul, Effect of substrate preparation on the perfection of GaP liquid phase epitaxy layers // J. Appl. Phys. 43 (6), p. 1186-1190 (1972).

5. T. Kawakubo, M. Okada, Optical and electron paramagnetic resonance studies on neutronirradiated GaP crystals // Phys. status solidi $(b)$ 106, p. 81-85 (1988).

6. G.R. Summers, E.A. Burke, P. Shapiro, S.R. Messenger, R.J. Walters, Damage correlations in semiconductors exposed to gamma, electron and proton radiations // IEEE Trans. Nucl. Sci. 40, p. 1372-1377 (1992).

7. A. Polity, Th. Abgarjan, R. Krause-Rehberg, Defects in electron irradiated GaP studied by positron lifetime spectroscopy // J. Appl. Phys. A 60, p. 541-544 (1995).

8. N. A. Vitovskij, The method of the determination of the charge of quasi-point clusters of impurities and defects in semiconductors and cluster distribution functions by charge // Fizika Tekhn. Poluprov. 16(5), p. 882-885 (1982) (in Russian).

9. P.G. Litovchenko, V.G. Makarenko, V.Ja. Opilat, V.P. Tartachnyk, I.I. Tychyna, Relaxation of the conductivity of irradiated GaP // Ukrain. Fizich. Zhurn. 33(3), p.367-390 (1988) (in Russian).

10. S.I. Radautcan, V.G. Makarenko, V.Ja. Opilat, V.P. Tartachnyk, I.I. Tychyna, Relaxation of the conductivity and radiation defect annealing in $\mathrm{GaP}$ irradiated by gamma-particles // Doklady $A N$ USSR. Ser. A 5, p. 50-53 (1988) (in Russian).

11. P. Litovchenko, D. Biselbo, A. Litovchenko, S. Kanevskyj, V. Opilat, M. Pinkovska, V. Tartachnik, R. Rando, P. Giubilabo, V. Khomenkov, Some features of current-voltage characteristics of irradiated GaP light diodes // Nucl. Instrum. Meth. Phys. Res. A552, p. 93-97 (2005).

12. L. Berman, Capacitive methods of semiconductor study. Nauka, Leningrad, 1972 (in Russian).

13. S.M. Sze, G. Gibbons, Avalanche breakdown voltages of abrupt and linearly graded p-n-junction in Ge, Si, GaAs and GaP // Appl. Phys. Lett. 8, p. III (1966).

14. K.S. Rzhevkin, Physical principles of semiconductor device operation. Moscow State University, Moscow, 1986, p. 255 (in Russian). 